\title{
$\bullet \bullet \bullet \bullet$ \\ Le développement des requêtes en langue étrangère : Comparaison entre le français L2, le finnois L1 et le français L1
}

TUULI HOLTTINEN 
L'auteure a soutenu sa thèse de doctorat Le développement des requêtes en langue étrangère: Comparaison entre le français L2, le finnois $L 1$ et le français L1 à la Faculté des Lettres, l'Université de Helsinki, le 26 novembre 2020. Le rapporteur était professeur Jean-Marc Dewaele (Birkbeck, University of London) et la présidente de la soutenance était professeure Mervi Helkkula. La thèse de doctorat est disponible à http://hdl.handle.net/10138/321003.

Mots-clés français, finnois, pragmatique de l'interlangue, compétence pragmatique, développement de langue étrangère, requête

\section{Introduction : l'acte de requête dans la vie quotidienne}

Dans la vie quotidienne, il serait difficile, voire impossible de s'en sortir sans l'aide des autres. Lorsque nous avons besoin que les autres agissent pour nous, nous faisons des requêtes. Les requêtes peuvent porter sur des actions très simples : nous faisons des requêtes par exemple dans les cafés et dans les magasins, dans le cadre du travail et des études ou quand nous faisons la cuisine en famille ou avec les amis. La formulation de requête dépend toutefois $d u$ contexte ; autrement dit, de la situation, de l'interlocuteur et de l'objet de la requête. Une requête destinée à un membre de famille pour qu'il accomplisse une tâche domestique n'est pas formulée de la même manière qu'une requête d'un service exceptionnel destinée à une connaissance ou à un supérieur hiérarchique. Cette activité langagière varie donc selon la situation.

Savoir formuler des requêtes de façon appropriée à chaque situation est une compétence acquise depuis l'enfance en interaction avec les parents et les proches. Par exemple, si un enfant demande une glace en criant « je veux une glace! ", ses parents peuvent lui demander " qu'est-ce qu'on dit pour demander ", ce qui conduit éventuellement à une reformulation telle que « est-ce que je peux avoir une glace s'il vous plaît ", ce qui satisfait les parents. La formulation des requêtes peut être difficile pour les locuteurs de langues étrangères parce qu'ils ne sont pas conseillés durant leur apprentissage comme le serait un enfant par son entourage dans sa langue maternelle. En outre, les formulations de requête typiques varient selon la langue et la culture, ce qui peut poser des problèmes aux apprenants de langue. J'en ai fait l'expérience... 
À la fin du collège, après avoir étudié le français deux heures par semaine pendant deux ans, je suis partie en France pour la première fois avec mes grands-parents pour tester mes connaissances. Pour le déjeuner, j'ai acheté des sandwichs dans une boulangerie près de l'hôtel. Je me rappelais qu'en classe de français, nous avions parlé de l'expression s'il vous plait ; il fallait l'ajouter à la fin de la commande pour être poli. Quand je me suis trouvée devant le comptoir, j'ai salué la vendeuse et formulé ma commande - mais sans s'il vous plaît. Je me demandais déjà à ce moment-là pourquoi je n’arrivais pas à prononcer cette phrase simple, même si je savais qu'il fallait l'utiliser.

Je ne suis pas la seule personne finnophone qui a vécu ce type d'expérience. De temps en temps, dans les journaux finlandais, on publie des articles qui parlent des Finlandais « impolis » qui, entre autres, ne savent pas dire please lorsqu'ils voyagent dans des pays anglophones. Les Finlandais ne sont toutefois pas plus impolis que les personnes venant d'autres langues-cultures - en revanche, la politesse linguistique en finnois diffère de celle en anglais ou en français. Par exemple, en finnois, il n'existe pas d'équivalent direct pour les phrases telles que please ou s'il vous plait, mais les requêtes perçues comme « polies » en finnois sont souvent formulées au conditionnel (Peterson et Vaattovaara $2014: 249$ ). Cette différence peut avoir un effet sur la façon dont les finnophones expriment la politesse lorsqu'ils utilisent des langues étrangères. Cette idée est à la base de ma thèse de doctorat dans laquelle j'ai étudié le développement des requêtes chez les locuteurs finnophones de français langue étrangère.

\section{Acte de requête et compétence pragmatique}

La fréquence et la nécessité des requêtes dans la vie quotidienne est l'une des raisons pour lesquelles elles ont fait l'objet d'étude dans bien des recherches linguistiques. Elles ont également été étudiées en pragmatique de l'interlangue, domaine auquel ma thèse de doctorat appartient. La pragmatique de l'interlangue examine les apprenants de langues différentes et la façon dont ils arrivent à savoir ce qu'il faut dire à des personnes différentes dans des situations variées. L'objectif est donc d'étudier les savoirs dont on a besoin pour utiliser la langue en contexte, c'est-à-dire la compétence pragmatique (Bachman 1990), appelée aussi compétence sociolin- 
guistique (Canale et Swain 1980 ; Canale 1983) ou compétence actionnelle et socioculturelle (Celce-Murcia et al. 1995).

D’une manière générale, les études sur les langues étrangères ont porté davantage sur le développement de la compétence grammaticale, par exemple, que sur celui de la compétence pragmatique. De plus, la majorité des études examinant la compétence pragmatique ont porté sur des locuteurs dont la langue première ou la langue cible est soit l'anglais, soit l'espagnol. La gamme des langues étudiées a donc été assez restreinte. Pour connaître les stades acquisitionnels généraux ainsi que les effets de la langue première et de la langue cible sur l'acquisition, il faut cependant examiner des groupes de locuteurs variés.

Pour contribuer à pallier cette lacune, j'ai examiné des apprenants finnophones de français dans ma thèse de doctorat. Plus précisément, j’ai étudié le développement de leur capacité à faire des requêtes en langue étrangère. Mon étude présente deux langues peu étudiées dans ce domaine : le finnois et le français. Ces langues présentent des différences étant donné qu'elles appartiennent à des familles de langue différentes : le finnois aux langues ouraliennes et le français aux langues indo-européennes. Elles présentent donc des différences liées à leur structure et à leur usage. Il faut toutefois noter qu'elles partagent aussi certains traits en tant que langues parlées en Europe (cf. Haspelmath 2001 ; Dahl 2008), ce qui rend leur comparaison fructueuse. Par exemple, il est possible de tutoyer ou vouvoyer son interlocuteur dans les deux langues, mais la manière dont les formes d'adresse sont visibles dans les structures diffère entre le finnois et le français. De plus, le choix entre le tutoiement et le vouvoiement dans des situations particulières varie considérablement entre ces langues (Havu et al. 2014).

Le finnois et le français sont donc suffisamment, mais pas excessivement différents pour être comparés. L'examen des apprenants finnophones de français peut nous fournir des informations qui corroborent les résultats des études antérieures en ce qui concerne les caractéristiques générales dans le développement de la compétence pragmatique. En outre, il est également possible d'obtenir des informations importantes sur les effets que la langue première des apprenants a sur ce développement. 


\section{Méthodologie et données}

Les études antérieures ont montré que le langage utilisé par les apprenants forme son propre système. Ce système est en permanence en transition et présente des caractéristiques propres à la langue première de l'apprenant et à la langue cible, mais aussi des caractéristiques particulières à cette « interlangue » (Selinker 1972 ; Ellis 1985 : 50-51 ; Kasper 2010 : 141). Pour examiner ces différents types de traits, j’ai comparé les requêtes produites par des apprenants finnophones de français à celles produites en finnois et en français langues premières.

Le corpus consiste en deux types de données. Premièrement, j’ai utilisé la méthode de Discourse Completion Test (v. p. ex. Ogiermann 2018) pour obtenir des requêtes orales. Cette méthode présente des descriptions de situations exigeant la production d'une requête, accompagnées d'une photo de l'interlocuteur imaginaire (Figure 1). Chaque informateur a donc lu la description et dit ensuite à voix haute ce qu'il ou elle dirait dans telle situation. La méthode m'a permis de comparer les requêtes des groupes d'informateurs différents dans les mêmes situations. J'ai enregistré les réactions de 90 locuteurs dans huit situations différentes. Ensuite, après ces réactions, les mêmes informateurs ont participé à un entretien rétrospectif pendant lequel ils ont réfléchi à leurs choix langagiers, par exemple au tutoiement et au vouvoiement. $\mathrm{Au}$ total, j'ai analysé 694 requêtes, dont 136 en finnois, 136 en français et 422 en français langue étrangère. 


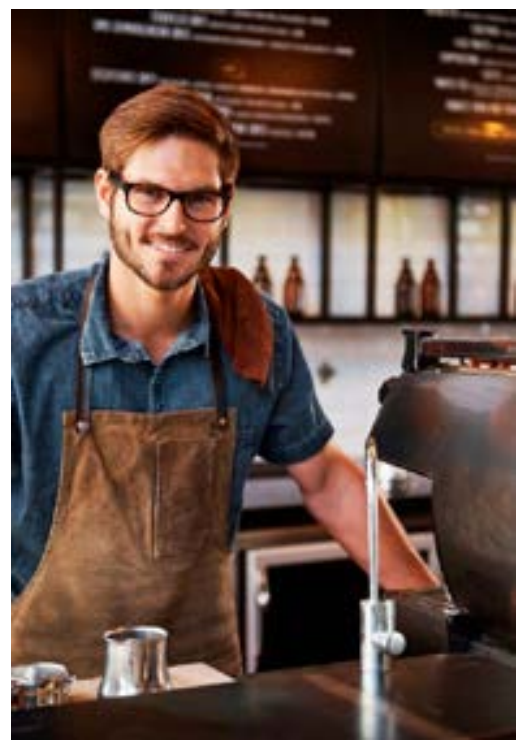

C'est le matin et vous vous sentez fatigué(e). Alors que vous vous dépêchez pour aller en cours, vous vous arrêtez en route pour acheter un café à emporter. Vous dites au vendeur du café :

Figure 1. Situation « café » présentée aux informateurs.

Tous les informateurs étaient des étudiants au moment de la collecte des données. Les locuteurs du groupe débutant $(n=21)$ participaient aux cours de français dans le Centre de langues à côté de leurs études. Quant aux locuteurs du groupe intermédiaire ( $n=15)$, ils étaient des étudiants de première année en philologie française, tandis que les locuteurs du groupe avancé ( $n=17)$ étaient des étudiants en master de la même filière. Les groupes de contrôle finnophone $(n=17)$ et francophone $(n=17)$ consistaient en des étudiants de diverses filières.

J'ai analysé certains traits dans les requêtes pour détecter les différences dans les formulations entre les langues examinées et à différents stades de l'acquisition. La classification adoptée se base sur celle de Blum-Kulka et al. (1989), employée et modifiée dans la majorité des études examinant les requêtes en L2 (p. ex. Flores Salgado 2011 ; Economidou-Kogetsidis 2012 ; Forsberg Lundell et Erman 2012). Par exemple, j'ai examiné les ouvertures pour savoir si les locuteurs saluent ou s'excusent avant de présenter la requête. Dans la requête à proprement parler, j'ai analysé la stratégie de requête qui peut être directe ou indirecte. Parmi les stratégies directes, on trouve par exemple 
l'emploi de l'impératif, " donne-moi une glace ", tandis que les requêtes formulées sous forme de questions sont considérées comme indirectes, par exemple « est-ce que tu peux me donner une glace ». Dans ces formulations, il est possible d'ajouter des éléments d'atténuation, tels que le marqueur de politesse s'il te plaît ou s'il vous plaît et le conditionnel.

J'ai également examiné les éléments qui précèdent ou suivent la requête à proprement parler, par exemple la manière dont les locuteurs justifient la requête. De plus, j’ai analysé la perspective : une requête peut être orientée vers le locuteur, " est-ce que je peux avoir une glace », vers son interlocuteur « est-ce que tu peux me donner une glace ", ou elle peut être impersonnelle, " est-ce qu'il serait possible d'avoir une glace ». Un autre facteur lié à la référence à l'interlocuteur est l'emploi des formes d'adresse, c'est-à-dire l'emploi $\mathrm{du}$ tutoiement et du vouvoiement et des formes nominales d'adresse telles que les prénoms et les titres. J'ai donc analysé toutes ces formes d'adresse dans les requêtes.

\section{Tendances dans le développement des requêtes en français L2}

J'ai examiné le développement des requêtes en français langue étrangère sous trois points de vue différents. Premièrement, j'ai examiné les requêtes à trois niveaux d'acquisition pour comprendre la façon dont les requêtes se développent en langue étrangère. Il est important de connaître les différentes trajectoires développementales par exemple pour pouvoir adapter l'enseignement aux besoins des apprenants.

Les résultats montrent que ces trajectoires varient selon le trait examiné : certains traits se développent de façon linéaire s'approchant de l'emploi en français langue première, tandis que d'autres présentent des développements non-linéaires. Dans le cas de certaines caractéristiques, les apprenants avancés sont plus loin de l'usage en langue cible que les apprenants débutants, ce qui veut dire que le développement s'écarte de la norme française. J'ai observé ce type de développement dans les ouvertures : les apprenants avancés ont sur-utilisé les salutations et sous-utilisé les excuses dans les ouvertures de requête, tandis qu'au niveau débutant, la distribution des ouvertures est plus proche de celle en français langue première. 
Deuxièmement, j'ai étudié l'influence de la langue première des apprenants, c'est-à-dire le finnois, sur les requêtes produites en français. De nombreuses études antérieures n’ont pas comparé systématiquement les requêtes produites à différents niveaux d'acquisition à celles produites en langue première et en langue cible. Dans ces études, il n’a pas été possible de déterminer les traits typiques pour les apprenants en général et ceux qui sont liés à l'influence de la langue première.

D’après les résultats, on peut observer l'influence du finnois dans de nombreuses caractéristiques dans les requêtes. Par exemple, l'absence de l'équivalent pour le marqueur de politesse s'il vous plaît en finnois suscite un sous-emploi de ce marqueur surtout au niveau débutant. D’un autre côté, il existe des traits qui ont tendance à être influencés par le finnois au niveau avancé, car dans ce cas, les apprenants possèdent les outils langagiers pour exprimer ce qu'ils veulent. Par exemple, les locuteurs du groupe avancé ont entouré les requêtes à proprement parler par des éléments divergeant de ceux utilisés en français langue première. À titre d'exemple, les apprenants avancés se sont présentés avant de faire la requête dans certaines situations où il fallait s'adresser à un inconnu (exemple 1), ce qu'ont fait aussi les locuteurs du groupe finnois langue première (exemple 2), contrairement à l'emploi en français L1 (exemple 3). Les apprenants avancés se sont donc servis aussi d'un usage propre à leur langue première en parlant français.

1. euh bonsoir en fait je suis votre voisine de dessous et euhm et en fait je suis en train de de réviser pour un examen et j'ai vraiment du mal à à me concentrer avec la musique donc est-ce qu'il serait possible de de baisser le son un peu (français L2, niveau avancé)

2. hei aa mä oon naapuri tosta alakerrasta aa tota oiskohan mitenkään mahollista et voisitte laittaa tota musiikkia hiljemmalle et se kuuluu aika hyvin tonne meidän asuntoon [bonjour je suis la voisine de dessous euhm serait-il éventuellement possible de baisser la musique on l'entend assez bien dans notre appartement] (finnois L1)

3. excuse-moi de te déranger mais je suis en train de préparer pour un examen très difficile et très important et ta musique est vraiment trop forte donc si tu pouvais la baisser ce serait très très cool (français L1) 
Troisièmement, j’ai comparé les requêtes des apprenants finnophones de français à celles examinées dans les études antérieures pour savoir comment ces locuteurs diffèrent des locuteurs ayant d'autres langues premières et étrangères. La caractéristique la plus frappante chez les apprenants finnophones de français se trouve dans l'emploi des marqueurs de politesse. La majorité des études antérieures a constaté un suremploi des marqueurs tels que please ou por favor en comparaison de l'usage en langue cible, probablement parce qu'il s'agit d'un moyen efficace d'exprimer la fonction de requête et la politesse à la fois. Comme je l'ai constaté, les apprenants finnophones de français s'opposent à cette tendance puisqu'ils ont du mal à utiliser s'il te plaît et s'il vous plaît suffisamment souvent. Ce marqueur ne devient routinier qu'au niveau avancé.

Les entretiens rétrospectifs montrent que les informateurs sont conscients de cette difficulté - le problème n’est donc pas lié au fait de ne pas connaître le marqueur de politesse français, mais à l'automatisation de son emploi. Dans ces entretiens, les informateurs des groupes de français langue étrangère ont également parlé des marqueurs de politesse dans les autres langues européennes. Ils expliquaient qu'ils n’étaient pas habitués à utiliser ces marqueurs à cause de l'absence de l'équivalent en finnois. Il est donc tout à fait naturel que les locuteurs finnophones de langues étrangères doivent s'exercer pour acquérir ces marqueurs.

Les résultats de ma thèse montrent que les apprenants finnophones de français cherchent à s'exprimer de façon claire et polie. Dans certains cas, cela conduit toutefois à des formulations de requête qui s'écartent de l'emploi en langue cible. Par exemple, les apprenants avancés ont utilisé clairement plus d'éléments entourant la requête pour la justifier que les locuteurs du groupe français langue première. De façon générale, les apprenants ont également utilisé des expressions plus explicites. Ils voulaient donc s'assurer d'être compris.

Une autre caractéristique qui illustre les efforts pour être poli se trouve dans l'emploi des formes d'adresse : les apprenants de français ont utilisé moins souvent le tutoiement et plus de formes nominales d'adresse que les locuteurs de français langue première. Certains apprenants ont vouvoyé l'interlocuteur même dans des situations où le tutoiement est le choix le plus général aussi bien en finnois qu'en français (exemples 4, 5 et 6). 
4. bonjour Maria euh excusez-moi mais euh pouvez-vous euh me voir les notes pour l'examen euh que arrive euh je je n'ai pas les notes moi-même et je dois euh apprendre quelque chose pour l'examen (français L2, niveau intermédiaire)

5. moro olisko sulla lainata sun muistiinpanoja pariks päiväks et mä olin pari viikkoo kipeenä ni mä saisin kopioitua ne omaan vihkooni [salut est-ce que tu pourrais me prêter tes notes pendant quelques jours car j'ai été malade pendant deux semaines pour que je puisse les copier dans mon cahier] (finnois L1)

6. excuse-moi est-ce que ça te dérangerait de me donner tes notes de cours parce que j'étais très malade et j'ai envie de réussir l'examen (français L1)

Ces locuteurs de français langue étrangère avaient donc appris qu'en français, le vouvoiement est plus commun qu'en finnois, et que l'emploi des formes nominales d'adresse est important en français, mais ils ont exagéré cette différence. Des études antérieures ont constaté que les apprenants de langues ont tendance à s'assurer d'être clairs et polis (Dewaele 2004 ; van Compernolle 2015). À cet égard, les apprenants finnophones de français sont similaires aux apprenants ayant d'autres langues premières et étrangères.

\section{Conclusion : fiabilité et implications}

Dans ma thèse de doctorat, j'ai examiné les requêtes élicitées, c'est-à-dire produites par les informateurs pour mon étude. Je me suis aussi servie des entretiens rétrospectifs. Ce type de combinaison de données s’est montré particulièrement fructueux : j'ai obtenu des informations comparables sur les formulations de requête utilisées par plusieurs groupes ainsi que des informations sur les perceptions que les locuteurs avaient à propos des requêtes et des situations de requête concernées. Si je n'avais examiné que les formulations de requête, j'aurais raté un point de vue important, celui des locuteurs. J'estime donc que la combinaison de méthodes est essentielle pour ce type d'étude.

Il faut toutefois souligner que j'ai rassemblé les requêtes dans des situations de recherche et non d'interactions authentiques. Je ne peux donc pas savoir si les locuteurs feraient des requêtes de la même manière dans des 
situations de communication réelles. Les études antérieures ont montré que les requêtes rassemblées par des méthodes de collecte différentes divergent dans une certaine mesure, bien que les caractéristiques générales soient similaires quelle que soit la méthode utilisée. Malgré cela, ma thèse a fourni des informations importantes sur les formulations estimées comme appropriées en finnois et en français et sur la manière dont les apprenants de français utilisent leurs ressources langagières pour formuler des requêtes.

Les implications pédagogiques de mon étude concernent notamment le langage métapragmatique qui pourrait être exploité plus en classe de langues pour aider les apprenants à comprendre les différentes manières d'agir dans des situations sociales. Pour apprendre les langues étrangères, il est utile de comparer les différents comportements et de discuter des facteurs extralinguistiques ayant un effet sur les choix langagiers. Par exemple, en finnois, le choix du pronom d'adresse est influencé notamment par l'âge de l'interlocuteur, tandis qu'en français d'autres facteurs tels que la distance sociale et la position hiérarchique entre les interlocuteurs sont également pertinents (cf. Havu 2006).

La chose la plus importante dans l'acquisition des langues est toutefois sans conteste l'utilisation de la langue cible en interaction avec les autres. La pratique des différentes formes linguistiques tout en tenant compte de leurs significations en contexte facilite l'automatisation de ces formes. Je pense toutefois que différents exercices métalinguistiques pourraient être utiles notamment dans l'acquisition de la compétence pragmatique. Par exemple, si quelqu'un m'avait dit en classe de langue au collège qu'en finnois, il n'existe pas d'équivalent direct pour s'il vous plaît, et que nous avions réfléchi à différentes façons langagières et non-langagières d'être poli, je n'aurais peut-être pas été aussi confuse devant le comptoir d'une boulangerie française.

Le but de mon étude n’a pas été de prétendre que les Finlandais sont impolis, mais de montrer que nos milieux linguistiques et culturels influencent la manière dont nous utilisons les langues étrangères. Il est tout à fait compréhensible qu'au début de l'apprentissage les ressources linguistiques soient restreintes, ce qui limite les possibilités d'expression. D’un autre côté, les apprenants avancés peuvent refuser de se conformer aux normes de la langue cible pour des raisons liées à l'identité, par exemple. 
Dans ma thèse de doctorat, j'ai analysé les formulations de requête utilisées par les apprenants finnophones de français et celles utilisées en finnois et en français langues premières. Je ne veux toutefois pas évaluer certaines formulations comme bonnes ou mauvaises. Une formulation de requête qui diffère de celle utilisée par les locuteurs langue première n'est pas automatiquement impolie. Je souhaite donc que les apprenants aient une attitude clémente envers leur parcours d'apprentissage et qu'ils apprécient les compétences déjà acquises. Les différences entre les langues et les cultures sont avant tout une richesse. $\mathbf{D}$

TUULI HOLTTINEN

UNIVERSITÉ DE HELSINKI 


\section{Références}

BACHMAN, Lyle F. 1990. Fundamental considerations in language testing. Oxford : Oxford University Press.

BLUM-KULKA, Shoshana - Juliane House - Gabriele Kasper éds. 1989. CrossCultural Pragmatics : Requests and Apologies. Norwood : Ablex.

CANALE, Michael 1983. From communicative competence to communicative language pedagogy. Language and Communication, éds. Richards, Jack C. - Richard W. Schmidt. New York : Longman. 2-27. CANALE, Michael - Merrill Swain 1980. Theoretical bases of communicative approaches. Applied Linguistics 1 : 1-47. https://doi.org/10.1093/ applin/I.1.1

CELCE-MURCIA, Marianne - Zoltan Dörnyei - Sarah Thurrell 1995. Communicative Competence : A Pedagogically Motivated Model with Content Specifications. Issues in Applied Linguistics 6 (2) : 5-35. https://doi.org/10.5070/L462005216 VAN COMPERNOLLE, Rémi A. 2015. Native and non-native perceptions of appropriateness in the French secondperson pronoun system. Journal of French Language Studies 25 (1) : 45-64. https://doi.org/ 10.1017/S0959269513000471

DAHL, Östen 2008. Kuinka eksoottinen kieli suomi on? Virittäjä 112 (4) : 545-559. https://journal.fi/virittaja/ article/view/40707

DEWAELE, Jean-Marc 2004. Enquête sur le choix (rapporté) du pronom d'allocution en français natif et non-natif. Franco-British Studies 33/34 : 46-54. https://cvc.cervantes.es/Lengua/ coloquio_paris/ponencias/pdf/ cvc_dewaele.pdf
ECONOMIDOU-KOGETSIDIS, Maria 2012. Modifying oral requests in a foreign language - the case of Greek Cypriot learners of English. Interlanguage Request Modification, éds.

Economidou-Kogetsidis, Maria - Helen Woodfield. Amsterdam - Philadelphia: John Benjamins. 163-201.

https://doi.org/10.1075/pbns.217.06eco

ELLIS, Rod 1985. Understanding Second

Language Acquisition. Oxford: Oxford University Press.

FLORES SALGADO, Elizabeth 2011. The Pragmatics of Requests and Apologies. Amsterdam - Philadelphia : John Benjamins. https://doi.org/ 10.1075/pbns.212

FORSBERG LUNDELL, Fanny - Britt Erman 2012. High-level requests : A study of long residency L2 users of English and French and native speakers. Journal of Pragmatics 44 (6-7) : 756-775. https:// doi.org/10.1016/j.pragma.2012.02.010 HASPELMATH, Martin 2001. The European Linguistic Area : Standard Average European. Language Typology and Language Universals, éds. Haspelmath, Martin - Wulf Oesterreicher Wolfgang Raible. Berlin : Mouton de Gruyter. 1492-1510. https://doi.org/ 10.1515/9783110194265-044 HAVU, Eva 2006. L'emploi des pronoms d'adresse en français : étude sociolinguistique et comparaison avec le finnois. Dialogic language use Dimensions du dialogismeDialogisches Sprachgebraudh, éds. Taavitsainen, Irma - Juhani Härmä Jarmo Korhonen. Helsinki : Société Néophilologique. 225-240.

HAVU Eva - Johanna Isosävi - Hanna Lappalainen 2014. Les stratégies d'adresse en finnois : comparaison 
entre deux types de corpus oraux

institutionnels. S’adresser à autrui : les

formes nominales d'adresse dans une

perspective comparative

interculturelle, éd. Catherine Kerbrat-

Orecchioni. Chambéry: Publication

Chambéry. 303-336.

KASPER, Gabriele 2010. Interlanguage

pragmatics. Variation and Change:

Pragmatic Perspectives, éds. Fried,

Mirjam - Jan-Ola Östman - Jef

Verschueren. Amsterdam -

Philadelphia : John Benjamins.

141-154. https://doi.org/

10.1075/hoph.6.11kas

OGIERMANN, Eva 2018. Discourse

completion tasks. Methods in

Pragmatics, éds. Jucker, Andreas H. -

Klaus P. Schneider - Wolfram Bublitz.

Berlin - Boston : De Gruyter. 229-256.

https://doi.org/

10.1515/9783110424928-009

PETERSON, Elizabeth - Johanna

Vaattovaara 2014. Kiitos and pliis : The relationship of native and borrowed politeness markers in Finnish. Journal of Politeness Research 10 (2) : 247-269. https://doi.org/10.1515/pr-2014-0011

SELINKER, Larry 1972. Interlanguage.

International Review of Applied

Linguistics in Language Teaching,

(10) : 209-231. https://doi.org/

$\underline{10.1515 / \mathrm{iral} .1972 .10 .1-4.209}$ 